\title{
Determining the Fundamental Unit of a Pure Cubic Field Given Any Unit
}

\author{
By N. S. Jeans and M. D. Hendy
}

\begin{abstract}
A number of algorithms which have been used to derive fundamental units for pure cubic fields suffer from the lack of absolute certainty that the units obtained are fundamental. We present here an algorithm which will correct this deficiency. Briefly, if $\eta$ is any nontrivial unit of a pure cubic field, then for some positive integer $N, \eta^{1 / N}$ will be a fundamental unit. Our method determines which of the real numbers $\eta^{1 / N}$ are integers of the field and, subsequently, will determine the coefficients of the fundamental unit. We illustrate the process with several numerical examples.
\end{abstract}

Introduction. In her paper [2], Marta Sved employs Szekeres' algorithm [3] to calculate a table of units of $F_{D}=Q\left(D^{1 / 3}\right)$, for $0<D<200, D$ cubefree. She asserts that these units are fundamental. However, she also makes the following comments: "Unfortunately, there is no general criterion known at present to decide whether a given unit of $F_{D}$ is fundamental or not, and all tables involve an element of uncertainty in this respect", and, "with one exception $(D=167)$ the computations produced at least two, but usually more, units for each $D$. In all cases, the units obtained were powers of the first one, and it can be confidently assumed that a fundamental unit has been found. Only in a few instances did the algorithm miss out on an occasional power of the fundamental unit. In most cases it was amply sufficient to compute the first 100 decimal digits of $\alpha$ and $\gamma$ (the nonrational generators for the integral basis) to produce a reasonable number of units. In the case of $D=167, \alpha$ and $\gamma$ were computed to 360 decimal places, yet even this accuracy was insufficient to obtain a second unit. At any rate the accuracy would have been sufficient to produce $\eta^{3 / 2}$ had the first calculated unit $\eta$ been the square of the fundamental unit."

Although Sved's optimism seems to be justified now on the basis of subsequent computations [1], there is no mathematical certainty that Szekeres' algorithm will always give the fundamental unit. Indeed, in several instances units were missed. In particular, in the case of $D=167$, no subsequent unit was obtained, which would have increased the confidence in the value of $\eta_{167}$.

Clearly, there is a need for a simple test to determine whether or not a unit is fundamental. Such a test would enhance the value of Szekeres' powerful algorithm

Received June 7, 1977; revised October 26, 1977.

AMS (MOS) subject classifications (1970). Primary 12 A30, 12 A45; Secondary 10F20, $12 \mathrm{~A} 34$.

Key words and phrases. Pure cubic fields, fundamental unit, Szekeres algorithm. 
to calculate units. This need has motivated us to develop such a test, which will not only determine whether or not a unit is fundamental, but will produce the fundamental unit when any nontrivial unit is tested.

Our approach is based on the relationship between the numerical value of a unit $\epsilon$, and its minimal polynomial. We are able to determine the coefficients of this polynomial, given $\epsilon$.

Any given unit $0<\epsilon<1$, will be a power of the fundamental unit. We calculate $\epsilon^{-1 / N}$ (as a real number, with $N \in Z^{+}$) and we then determine values which will be the coefficients of the minimal polynomial for $\epsilon^{1 / N}$ if it is a cubic integer. These values are integral if and only if $\epsilon^{1 / N}$ is a cubic integer and, hence, also a unit. We know $\epsilon^{1 / N}$ is a fundamental unit for some $N \in Z^{+}$(possibly $N=1$ ), and we will show that only a small number of integers $N$ need be considered. Hence, we are able to determine conclusively which root of $\epsilon$ is fundamental. We also illustrate our algorithm with several numerical examples, one of which verifies Sved's unit $\eta_{167}$ as fundamental.

Our algorithm used in conjunction with Szekeres' algorithm would not only be conclusive, but would also be more efficient than the direct use of Szekeres' algorithm described by Sved. In that application, Sved, having initially obtained a unit, continues to calculate units with larger coefficients. This involves working with numbers of increasing precision. As in many cases this involves computations using multiprecision arithmetic, these further computations may be very expensive. Also, she makes no use of the unit already obtained.

Having obtained a unit, our algorithm would immediately be applied to the numerical value of this unit, and computation would involve a sequence of numbers in which the need for precision decreased steadily. For example, for $D=167$, Sved continues her calculations with numbers of up to 360 significant digits; whereas, with our algorithm the computations would commence with numbers of 100 significant digits, with significance decreasing at each step. (In the final step, only 10 significant digits are necessary.) Thus, having obtained a unit, we work in the 'opposite direction' as far as precision is concerned.

Conditions for a Real Number to be a Cubic Unit. Let $p, q$ be two distinct relatively prime squarefree integers with $p>0, q>0$ then $d=p q^{2}>1$ is cubefree. Let $\alpha=d^{1 / 3}$. The integers of $Q(\alpha)$ are the numbers:

$$
\beta=\left(x+y\left(p q^{2}\right)^{1 / 3}+z\left(p^{2} q\right)^{1 / 3}\right) / 3,
$$

where $x, y, z \in Z$ and $x \equiv y \equiv z \equiv 0(\bmod 3)$ is $p \not \equiv \pm q(\bmod 9)$, (Type $\mathrm{I})$ or $x \equiv$ $p y \equiv q z(\bmod 3)$ if $p \equiv \pm q(\bmod 9)$, (Type II). If $\beta \in Z$, then it satisfies the minimal polynomial:

$$
\beta^{3}-x \beta^{2}+\frac{1}{3}\left(x^{2}-p q y z\right) \beta-N(\beta)=0,
$$


(3) $N(\beta)=\left(x^{3}+p q^{2} y^{3}+p^{2} q z^{3}-3 x y z p q\right) / 27$,

$$
\begin{aligned}
& =\beta\left(\left(x^{2}-p q y z\right)+\left(p z^{2}-x y\right)\left(p q^{2}\right)^{1 / 3}+\left(q y^{2}-x z\right)\left(p^{2} q\right)^{1 / 3}\right) / 9, \\
& =\beta\left(\left(x-y\left(p q^{2}\right)^{1 / 3}\right)^{2}+\left(y\left(p q^{2}\right)^{1 / 3}-z\left(p^{2} q\right)^{1 / 3}\right)^{2}+\left(z\left(p^{2} q\right)^{1 / 3}-x\right)^{2}\right) / 18
\end{aligned}
$$

As the second term of (4) is positive definite, $\beta$ has the same sign as its norm.

We now list some properties of units of $Q(\alpha)$.

LEMMA 1. Let $\mu=\left(x+y\left(p q^{2}\right)^{1 / 3}+z\left(p^{2} q\right)^{1 / 3}\right) / 3>1$ be a unit of $Q(\alpha)$.

Then:
(A) $N(\mu)=1$;
(B) $x, y, z$ are each nonzero;
(C) $x, y\left(p q^{2}\right)^{1 / 3}, z\left(p^{2} q\right)^{1 / 3} \geqslant 1$;
(D) $\mu>3$.

Proof. (A) $N(\mu)= \pm 1, \mu>0$, so from (4), $N(\mu)>0$. Thus, $N(\mu)=1$.

(B) Trivially, if any two of $x, y, z$ are zero, then $\mu=1,\left(p q^{2}\right)^{1 / 3}$ or $\left(p^{2} q\right)^{1 / 3}$, which cannot hold. Suppose one of $x, y$ or $z$ is zero, then by (1) $x \equiv y \equiv z \equiv 0$ $(\bmod 3)$. Let $x_{0}=x / 3, y_{0}=y / 3, z_{0}=z / 3$. If $x=0, N(\mu)=y_{0}^{3} p q^{2}+z_{0}^{3} p^{2} q$, a multiple of $p q$, but $N(\mu)=1$, a contradiction. Hence $x \neq 0$. If $y=0, N(\mu)=x_{0}^{3}+$ $z_{0}^{3} p^{2} q=1$. As $x_{0}^{3}, z_{0}^{3} p^{2} q$ are nonzero, they must differ in sign; $\Rightarrow x_{0}^{3} z_{0}^{3} p^{2} q<0$,

$$
\begin{gathered}
\Rightarrow x_{0} z_{0}\left(p^{2} q\right)^{1 / 3}<0 . \\
\mu>1 \Rightarrow \mu^{3}=x_{0}^{3}+z_{0}^{3} p^{2} q+3 x_{0} z_{0}\left(p^{2} q\right)^{1 / 3}\left[x_{0}+z_{0}\left(p^{2} q\right)^{1 / 3}\right] \\
=1+3 x_{0} z_{0}\left(p^{2} q\right)^{1 / 3} \mu>1 .
\end{gathered}
$$

$\Rightarrow x_{0} z_{0}\left(p^{2} q\right)^{1 / 3}>0$, contradicting (5). Thus $y \neq 0$. If $z=0$, by symmetry we obtain a similar contradiction.

(C) Let $a>b>c$ be a permutation of $x / 3, y\left(p q^{2}\right)^{1 / 3} / 3$, and $z\left(p^{2} q\right)^{1 / 3} / 3$. (Equality cannot hold as $1,\left(p q^{2}\right)^{1 / 3}$ and $\left(p^{2} q\right)^{1 / 3}$ are rationally independent.)

$$
\mu=a+b+c>1 \text {. }
$$

$$
\begin{aligned}
0<\mu^{-1} & =\left(\left(x^{2}-p q y z\right)+\left(p z^{2}-x y\right)\left(p q^{2}\right)^{1 / 3}+\left(q y^{2}-x z\right)\left(p^{2} q\right)^{1 / 3}\right) / 9 \\
& =\left(a^{2}-b c\right)+\left(b^{2}-c a\right)+\left(c^{2}-a b\right)<1, \quad \text { and } \\
N(\mu) & =\left(x\left(x^{2}-p q y z\right)+p q y\left(q y^{2}-x z\right)+p q z\left(p z^{2}-x y\right)\right) / 27 \\
& =a\left(a^{2}-b c\right)+b\left(b^{2}-c a\right)+c\left(c^{2}-a b\right)=1
\end{aligned}
$$

Consider the six terms $a, b, c, a^{2}-b c, b^{2}-c a, c^{2}-a b$. We know from (B) that $a, b, c$ are nonzero. If two of the other terms were zero, say $a^{2}=b c$ and $b^{2}=c a$, then

$$
a^{4}=b^{2} c^{2}=a c^{3} \Rightarrow a=b=c
$$

which cannot hold. For fields of Type I, $p^{2} \not \equiv q^{2}(\bmod 9)$, then $a, b, c, a^{2}-b c, b^{2}$ $-c a, c^{2}-a b$ are integral multiples of $1,\left(p q^{2}\right)^{1 / 3}$ or $\left(p^{2} q\right)^{1 / 3}$. Hence, at least one of the summands in both (8) and (10) must be negative. 
For fields of Type II, $p^{2} \equiv q^{2}(\bmod 9)$, and from an inspection of cases $|p q| \geqslant 10$. $a, b, c, a^{2}-b c, b^{2}-c a, c^{2}-a b$ are integral multiples of $1 / 3,\left(p q^{2}\right)^{1 / 3} / 3,\left(p^{2} q\right)^{1 / 3} / 3$, so at least one summand in (8) must be negative. (If one term is zero, then from (1), the other two are integral multiples of $1,\left(p q^{2}\right)^{1 / 3},\left(p^{2} q\right)^{1 / 3}$.) Suppose all the terms in (9) are nonnegative, then (9) may be rewritten as

$$
27=x\left(x^{2}-p q y z\right)+|p q| A,
$$

where $A \in Z$. We note that $x\left(x^{2}-p q y z\right) \equiv 0(\bmod 3)$. Thus, $p q A \equiv 0(\bmod 3)$. But $p q \equiv 0(\bmod 3)$ so $A \equiv 0(\bmod 3)$. $A \neq 0$, for otherwise $\mu=1$, and we have assumed the terms in (9) to be nonnegative. Hence, $A \geqslant 3 \Rightarrow|p q| A \geqslant 30$, contradicting (11). Hence, at least one term in (9) is negative.

Hence, for fields of both Types I, and II, at least one term in both (8) and (10) is negative.

By (6) $a>0$. Suppose $a>0>b>c$, then $b^{2}>a c, c^{2}>a b$, and as one term in (8) must be negative $a^{2}<b c \Rightarrow a^{2}<2 b c<(b+c)^{2} \Rightarrow a<-(b+c) \Rightarrow a+b+$ $c=\mu<0$, a contradiction.

Suppose $a>b>0>c$, then $a^{2}>b c, b^{2}>c a$, and as one term in (8) must be negative $c^{2}<a b$. Now $c^{2}<a b \Rightarrow c\left(c^{2}-a b\right)>0 \Rightarrow$ all terms in (10) are positive, a contradiction. Thus, $a>b>c>0$. Hence, $x, y\left(p q^{2}\right)^{1 / 3}, z\left(p^{2} q\right)^{1 / 3} \geqslant 1$.

(D) We have $\mu=\left(x+y\left(p q^{2}\right)^{1 / 3}+z\left(p^{2} q\right)^{1 / 3}\right) / 3$, and by (C), each term is positive.

For fields of Type I $p^{2} \not \equiv q^{2}(\bmod 9)$, then $\mu>3$ since $x \equiv y \equiv z \equiv 0(\bmod 3)$. For fields of Type II $p^{2} \equiv q^{2}(\bmod 9)$. If $|p q|>16,|p q|^{1 / 3}>4^{2 / 3} \Rightarrow|p|^{1 / 3}+$ $|q|^{1 / 3}>2.4^{1 / 3} \Rightarrow y\left(p q^{2}\right)^{1 / 3}+z\left(p^{2} q\right)^{1 / 3}=|p q|^{1 / 3}\left(y p^{1 / 3}+|z q|^{1 / 3}\right)>$ $4^{2 / 3} 2.4^{1 / 3}=8, \Rightarrow \mu>(1+8) / 3=3$.

There are only four fields of Type II with $|p q| \leqslant 16$, namely those for which

$$
\begin{aligned}
& d=10,100, \quad \text { with } p q=10 \\
& d=28,98, \quad \text { with } p q=14 .
\end{aligned}
$$

In these cases, the smallest units $>1$ are $\mu \doteqdot 23.3$ for $d=10,100$ and $\mu \doteqdot 5.2$ for $d=28,98[1]$. Hence, $\mu>3$ in all cases.

We now consider the relationship between the numerical value of the unit and its minimal polynomial. For this we consider the reciprocal $\epsilon=1 / \mu$ of the unit above.

Lemma 2. Let $\epsilon=\mu^{-1}=\left(l+m\left(p q^{2}\right)^{1 / 3}+n\left(p^{2} q\right)^{1 / 3}\right) / 3<1$. Then $\epsilon$ satisfies the minimal polynomial:

$$
\epsilon^{3}-l \epsilon^{2}+f \epsilon-1=0
$$

where $f=\left(l^{2}-p q m n\right) / 3$.

For $|p q|>8$,

$$
f>\frac{1}{6} l^{2},
$$

$$
|f-\mu|<2.75 \epsilon^{1 / 2} \text {. }
$$


Proof. (A) If $m n=0, f=l^{2} / 3>l^{2} / 6$. If $m n \neq 0,|m|,|n| \geqslant 1$.

$$
\begin{aligned}
(l-3 \epsilon)^{2} & =\left(m\left(p q^{2}\right)^{1 / 3}+n\left(p^{2} q\right)^{1 / 3}\right)^{2} \\
& =m^{2} p^{2 / 3} q^{4 / 3}+n^{2} p^{4 / 3} q^{2 / 3}+2 m n p q
\end{aligned}
$$

Now $m^{2} p^{2 / 3} q^{4 / 3}>|p q|^{1 / 3}\left|m p^{1 / 3} q^{2 / 3}\right|, n^{2} p^{4 / 3} q^{2 / 3}>|p q|^{1 / 3}\left|n p^{2 / 3} q^{1 / 3}\right|$, and as $|p q|>8$,

$$
\begin{aligned}
(l-3 \epsilon)^{2} & >2\left|m p^{1 / 3} q^{2 / 3}+n p^{2 / 3} q^{1 / 3}\right|+2 m n p q \\
& =2|l-3 \epsilon|+2 m n p q
\end{aligned}
$$

Hence,

$$
\begin{aligned}
l^{2} & >2|l-3 \epsilon|+2 m n p q+6 l \epsilon-9 \epsilon^{2} \\
& =2|l-3 \epsilon|+2 m n p q+6 \epsilon(l-3 \epsilon)+9 \epsilon^{2} \\
& >(2-6 \epsilon)|l-3 \epsilon|+2 m n p q .
\end{aligned}
$$

As $0<\epsilon<1 / 3$ (Lemma 1(D)), $(2-6 \epsilon)|l-\epsilon|>0$, so $l^{2}>2 m n p q \Rightarrow f=$ $\left(l^{2}-m n p q\right) / 3>l^{2} / 6$.

(B) From (12) we have $\epsilon^{2}-l \epsilon+f-\mu=0$, so

$$
\begin{aligned}
\mu- & \epsilon^{2}=f-l \epsilon>\frac{1}{6} l^{2}-l \epsilon=(l-3 \epsilon)^{2} / 6-3 \epsilon^{2} / 2, \\
& \Rightarrow(l-3 \epsilon)^{2}<6\left(\mu-\epsilon^{2}\right)+9 \epsilon^{2}=6\left(\mu+\epsilon^{2} / 2\right)=6 \mu\left(1+\epsilon^{3} / 2\right), \\
& \Rightarrow|l-3 \epsilon|<6^{1 / 2} \mu^{1 / 2}\left(1+\epsilon^{3} / 2\right)^{1 / 2} \\
& \Rightarrow|l-\epsilon|<6^{1 / 2} \mu^{1 / 2}\left(1+\epsilon^{3} / 2\right)^{1 / 2}+2 \epsilon \\
& \Rightarrow|f-\mu|=\left|l \epsilon-\epsilon^{2}\right|<6^{1 / 2} \epsilon^{1 / 2}\left(1+\epsilon^{3} / 2\right)^{1 / 2}+2 \epsilon^{2} .
\end{aligned}
$$

Now as $0<\epsilon<1 / 3,6^{1 / 2}\left(1+\epsilon^{3 / 2}\right)^{1 / 2}+2 \epsilon^{3 / 2}<2.75$, so we have

$$
|f-\mu|<\left(6^{1 / 2}\left(1+\epsilon^{3} / 2\right)^{1 / 2}+2 \epsilon^{3 / 2}\right) \epsilon^{1 / 2}<2.75 \epsilon^{1 / 2} .
$$

We make use of result (B) for our theorem. What we wish to determine is whether or not the real number $\epsilon_{N}=\epsilon^{1 / N}$ is a unit of $Q(\alpha)$. This is equivalent to determining whether or not it satisfies a monic integral cubic polynomial with constant term -1 .

THEOREM. $d=p q^{2}>1$ is cubefree, with $p, q$ squarefree integers and $|p q|>$ 8. For $\theta$ irrational let $\{\theta\}$ represent the closest integer to $\theta$.

Given $\epsilon$ a unit of $Q\left(d^{1 / 3}\right), 0<\epsilon<1$, set $\epsilon_{N}=\epsilon^{1 / N}$, for some positive integer $N$.

(A) For $\epsilon_{N}<1 / 25, \epsilon_{N}$ is a unit of $Q\left(d^{1 / 3}\right)$

$$
\Longleftrightarrow \epsilon_{N}^{3}-l \epsilon_{N}^{2}+f \epsilon_{N}-1=0,
$$

where $f=\left\{\epsilon_{N}^{-1}\right\}, l=\left\{f \epsilon_{N}^{-1}-\epsilon_{N}^{-2}\right\}$.

(B) $\epsilon_{N}$ is a unit of $Q\left(d^{1 / 3}\right) \Rightarrow$

$$
\left|f-\epsilon_{N}^{-1}\right|<2.75 \epsilon_{N}^{1 / 2} .
$$


Notes. For $\epsilon_{N}>1 / 25$ each of the several (up to 4) values of $f$ satisfying (15) can be tested in Eq. (14). Then $\epsilon_{N}$ is a unit $\Longleftrightarrow$ one of these values satisfies (14).

In practice, for $\epsilon_{N}$ small, condition (B) screens out most $\epsilon_{N}$ tested as not being units. The probability that this test is "accidentally" satisfied is low.

Proof. For $\epsilon_{N}<1 / 25,\left|f-\epsilon_{N}^{-1}\right|<6^{1 / 2} \epsilon_{N}^{1 / 2}\left(1+\epsilon_{N}^{3} / 2\right)^{1 / 2}+2 \epsilon_{N}^{2}<0.5$, from (13) so a unique value of $f$ is determined.

If $\epsilon_{N}$ is a unit of $Q\left(d^{1 / 3}\right)$, then by Lemma 2(B) it satisfies a minimal polynomial of the form

$$
\epsilon_{N}^{3}-l \epsilon_{N}^{2}+f \epsilon_{N}-1=0
$$

where $l-f \epsilon_{N}^{-1}+\epsilon_{N}^{-2}=\epsilon_{N}<1 / 25$, so $l=\left\{f \epsilon_{N}^{-1}-\epsilon_{N}^{-2}\right\}$. If $\epsilon_{N}$ satisfies an equation of the form

$$
\epsilon_{N}^{3}-l \epsilon_{N}^{2}+f \epsilon_{N}-1=0
$$

with $f=\left\{\epsilon_{N}^{-1}\right\}>0$, then $\epsilon_{N}$ is a nonrational unit of some cubic field $K$. Hence $\epsilon=$ $\left(\epsilon_{N}\right)^{N} \in K \cap Q\left(d^{1 / 3}\right), \Rightarrow K \subset Q\left(d^{1 / 3}\right) \Rightarrow \epsilon_{N}$ is a unit of $Q\left(d^{1 / 3}\right)$.

Fundamental Unit Algorithm. Given $\mu>1$ a unit of the pure cubic field $Q\left(d^{1 / 3}\right)$ where $d=p q^{2}>1$ is cubefree, with $p, q$ squarefree integers and $|p q|>8$ :

1. Set

$$
\begin{aligned}
L & =1+\left(p q^{2}\right)^{1 / 3}+\left(p^{2} q\right)^{1 / 3} & & \text { for fields of Type I, } \\
& =\left(1+\left(p q^{2}\right)^{1 / 3}+\left(p^{2} q\right)^{1 / 3}\right) / 3 & & \text { for fields of Type II. }
\end{aligned}
$$

( $L$ is a lower bound on the size of any unit $\eta>1$ of $Q\left(d^{1 / 3}\right)$, (Lemma 1(C)).)

2. Set $r=2$.

3. Set $N=[\log \mu / \log L+1]$ (i.e. $N$ least integer such that $\mu^{1 / N}<L$ ).

4. If $r \geqslant N$ go to 10 .

5. Set $\mu(r)=\mu^{1 / r}$.

6. If $\nexists f \in Z$ such that $|f-\mu(r)|<2.75(\mu(r))^{-1 / 2}$, go to 8 .

7. If $\exists f \in Z$ such that $|f-\mu(r)|<2.75(\mu(r))^{-1 / 2}$ and $l=f \mu(r)-\mu(r)^{2}+$ $\mu(r)^{-1} \in Z$, go to 9 .

8. Increment $r$ to the next largest prime and return to 4 .

9. Reset $\mu=\mu(r)$ and return to 3 .

10. The current value of $\mu$ is fundamental. Stop.

Notes. (a) At step 7, for $\mu(r)>25$ only one such value of $f$ can exist. For smaller $\mu$, up to 4 values of $f$ could satisfy the condition of step 6 . In practice, for $\mu(r)>1000$, it is rare for $\mu(r)$ to satisfy step 6 and yet not be a unit. This becomes the more practical test for large $\mu(r)$.

(b) Obviously, only prime values $r$ need be considered.

(c) If $\mu(r)$ is fundamental, the coefficients of $\mu(r)^{-1}$ can be determined as follows from $f$ and $l$. $\mu(r)^{-1}=\left(l+m\left(p q^{2}\right)^{1 / 3}+n\left(p^{2} q\right)^{1 / 3}\right) / 3$, where $m n=$ $\left(l^{2}-3 f\right) / p q$. 
Practical Use of the Algorithm. In practice, we need to be able to calculate real numbers to approximately $\left[\log _{10} \mu+7\right]$ decimal digits. (In the case $\mu(2)$ satisfies the test at step 6.) So, for large $\mu$, multiprecision arithmetic computer programs are necessary (e.g. for the field $Q\left(167^{1 / 3}\right), \mu \doteqdot 10^{96}$ ). The calculation of $\mu(r)$ is performed using Newton-Raphson iteration for $r$ th roots. $\mu(r)$ need only be calculated to approximately $\left[\left(3 \log _{10} \mu(r)\right) / 2+2\right]$ digits in order to satisfactorily perform the test at step 6.

Since the calculation at step 7 involves irrational numbers, a conclusive result cannot be obtained no matter how many decimal digits are calculated. We can overcome this small problem by calculating $l$, at step 7, to (say) 5 decimal place accuracy. This requires calculating $\mu(r)$ to [2 $\left.\log _{10} \mu(r)+7\right]$ decimal digits. If $l$ differs from an integer by no more than $10^{-4}$, we would have reasonable confidence that $\mu(r)$ is indeed a cubic integer, and final verification would depend on the existence of integers, $m$ and $n$, determined by the simultaneous equations given in Note (c) above, and satisfying $l^{3}+p q^{2} m^{3}+p^{2} q n^{3}-3 p q l m n=27$. Almost all tests derive a nonintegral value of $l$, so this final verification is rarely needed. Indeed, no example of $\mu(r)>1000$ has been found to satisfy this condition other than when $\mu(r)$ was a unit.

Although multiprecision arithmetic is needed for large $\mu$, it is only necessary for small primes $r$. For example, with $d=167$, all primes from 2 to 61 must be considered, but for $r=13$ onwards double precision arithmetic was sufficient. The majority of the computing time is involved in the computations with $r=2,3,5,7$ and 11. For $\mu(r)<25$, several values of $f$ must be considered, but with single precision accuracy this involves little time.

Examples. We give three examples showing how we determined whether or not three given units are fundamental. The calculations were done on a Burroughs B6700 computer. We take the smallest unit $\mu>1$ to be the fundamental unit.

Example 1: $\mu=x+y\left(23^{1 / 3}\right)+z\left(23^{2 / 3}\right)$ is a unit of $Q\left(23^{1 / 3}\right)$, where

$$
\begin{array}{rrrr}
x= & 251401129 & 6271379187 & 9829592761 \\
7258440514 & 3510195116 & 6439999601 \\
y= & 88401156 & 3861048459 & 5086024628 \\
7875956789 & 8501378224 & 5618425660 \\
z= & 31084842 & 2280000275 & 0405930152 \\
0668641067 & 2496635313 & 4343732220 \\
\mu & & & \\
& & & \\
& 754203388 & 8814137563 & 9488778285 \\
1775321543 & 0530585349 & 9319998803 \\
.0000000000 & 0000000000 & 0000000004 \\
\doteqdot 7.542 \times 10^{58} & &
\end{array}
$$

(This $\mu$ is obtained by taking the reciprocal of the sixth power of the unit given by Sved [2].)

Applying the algorithm, we obtain: 


$$
\begin{aligned}
L= & 11.93, \quad N=55 . \\
\mu(2) \doteqdot & 2746276367 \quad 8869134462 \quad 7557332202 \\
& \cdot 9999999999 \quad 9999815705 \\
\text { and } \quad & 2.75(\mu(2))^{-1 / 2} \doteqdot 5.247 \times 10^{-15}
\end{aligned}
$$

Thus, the test at step 6 is positive, so we take

$$
f=27462763678869134462 \quad 7557332203
$$

and calculate

$$
l=50612 \quad 3590417203 \cdot 00000 \quad \text { (to } 5 \text { decimal places). }
$$

Solving the equations in Note (c) gives us $m, n$ such that $l^{3}+23 m^{3}+529 n^{3}-$ $69 l m n=27$. Thus $l, m, n$ are the coefficients of the unit $(\mu(2))^{-1}$, i.e. $\mu(2)$ is a unit.

We start again, replacing $\mu$ with $\mu(2)$. We recalculate $N$ i.e. $N=28$

$$
\mu(2) \doteqdot 524049269428640 \cdot 024917 \text {, }
$$

clearly not a unit, since the test at step 6 is negative.

$$
\mu(3) \doteqdot 6500020803 \cdot 0000191071
$$

and $2.75(\mu(3))^{-1 / 2} \doteqdot 0.00003411$.

The test at step 6 is positive, so we take

$$
f=6500020803 \text {, }
$$

and calculate

$$
l=-124197.00000 \quad \text { (to } 5 \text { decimal places). }
$$

Solving the equations in Note (c) gives us

$$
m=-9480, \quad n=18690 \text {, }
$$

and since $l^{3}+23 m^{3}+529 n^{3}-69 l m n=27$, we conclude that $[\mu(3)]^{-1}=$ $\left(l+m\left(23^{1 / 3}\right)+n\left(23^{2 / 3}\right)\right) / 3$, and hence $\mu(3)$, are units of $Q\left(23^{1 / 3}\right)$.

Replacing $\mu$, with $\mu(3)$, we start again. $N$ now takes the value 10 .

$$
\begin{gathered}
\mu(3) \doteqdot 1866.257 \text { and } 2.75(\mu(3))^{-1 / 2} \doteqdot 0.0637 \quad * \\
\text { so } \mu(3) \text { is not a unit. } \\
\mu(5) \doteqdot 91.745 \text { and } 2.75(\mu(5))^{-1 / 2} \doteqdot 0.287 .
\end{gathered}
$$

Therefore, taking $f=92$, we calculate

$$
\begin{aligned}
l & \doteqdot 23.39536 \text { and so } \mu(5) \text { is not a unit. } \\
\mu(7) & \doteqdot 25.225,2.75(\mu(7))^{-1 / 2} \doteqdot .548
\end{aligned}
$$

Take $f=25$, and calculate

$$
l \doteqdot-5.650 \text { i.e. not a unit. }
$$

Since the next prime is greater than 10 , we stop. 


\begin{tabular}{|c|c|c|c|c|}
\hline$r$ & $\mu(r)$ & $2.75(\mu(r))^{-1 / 2}$ & f & $\ell$ \\
\hline 17 & $431409 \cdot 595$ & 0.004 & - & \\
\hline 19 & $110088 \cdot 893$ & 0.008 & - & \\
\hline 23 & 14619.029 & 0.022 & - & \\
\hline 29 & $2010 \cdot 070$ & 0.061 & - & \\
\hline 31 & 1230.554 & 0.078 & - & \\
\hline 37 & 388.153 & 0.139 & - & \\
\hline 41 & $216 \cdot 979$ & 0.186 & 217 & $4 \cdot 559$ \\
\hline 43 & $168 \cdot 945$ & 0.211 & 169 & $9 \cdot 180$ \\
\hline 47 & $109 \cdot 182$ & 0.263 & 109 & $-19 \cdot 878$ \\
\hline 53 & $64 \cdot 182$ & 0.343 & 64 & $-11 \cdot 705$ \\
\hline 59 & 42.035 & 0.424 & 42 & $-1 \cdot 452$ \\
\hline 61 & $37 \cdot 185$ & 0.451 & 37 & -6.888 \\
\hline
\end{tabular}

(Calculations have been chopped to three decimal places.)

We conclude that

$$
\mu \doteqdot 6500020803.0000191071 \text { is the fundamental unit. }
$$

$\left({ }^{*} \mu(2)\right.$ need not be tested, since the test at (16) is negative.)

Example 2. From Sved [2], a unit in $Q\left(167^{1 / 3}\right)$ is

$$
\begin{aligned}
& \mu \doteqdot \quad 621050 \quad 0640303132 \quad 6161103562 \\
& \begin{array}{lll}
8827556350 & 7362478867 & 5341597327
\end{array} \\
& \begin{array}{llll}
4816722532 & 9679837287 & 8103562731
\end{array} \\
& 1528000987 \cdot 0000000000 \quad 0000000000 \\
& 0000000000 \quad 0000000000 \quad 0000000200 \\
& 1896095838 \\
& \mu \doteqdot 6.21 \times 10^{95} \text {. }
\end{aligned}
$$

(This is the reciprocal of the unit given by Sved.)

$$
\begin{array}{rrrr}
L & \doteqdot 36.84 \text { and so } N=62 & \\
\mu(2) & \doteqdot \quad 78806729 & 6637992244 & 0050104080 \\
3056469673 & 0335831406 & 2371560251 .
\end{array}
$$

Clearly, the test at step 6 is negative, i.e. $\mu(2)$ is not a unit. 


$$
\begin{aligned}
& \mu(3) \doteqdot \quad 85 \quad 3183020141 \quad 7547364081 \\
& 5443829075 \cdot 5805448903 \text {, } \\
& \text { clearly not a unit. } \\
& \mu(5) \doteqdot 1440871735 \quad 3864111266 \cdot 2999492424 \text {, } \\
& \text { not a unit } \\
& \mu(7) \doteqdot 48387409603319 \cdot 6430345357 \text {, } \\
& \text { not a unit. } \\
& \mu(11) \doteqdot 511053133 \cdot 5546616359 \text {, } \\
& \text { not a unit. } \\
& \mu(13) \doteqdot 23372332 \cdot 0124097584 \text {, } \\
& \text { not a unit. }
\end{aligned}
$$

The remaining calculations are given in the table above with $l$ being calculated only for the cases in which the test at step 6 is positive.

Therefore, we can conclude that $\mu$ is fundamental.

Example 3. From Sved [2] a unit in $Q\left(177^{1 / 3}\right)$ is

$$
\begin{array}{rrrr}
\mu \doteqdot & 458999 & 9459610388 & 1491148208 \\
5596864397 & 0592498560 & 1356525509 \\
5531283145 & 7224626001 & 8132349978 \\
.9999999999 & 9999999999 & 9999999999 \\
& 9999999999 & 9970485063 & \\
\doteqdot 4.59 \times 10^{85} & &
\end{array}
$$

Using the algorithm, we obtain

$$
\begin{aligned}
& L \doteqdot 38 \cdot 14, \quad N=55 \\
& \mu(2) \doteqdot \quad 677 \quad 4953475567 \quad 4803446400 \\
& 17961018816158301743 \cdot 1601593529 \\
& 2.75(\mu(2))^{-1 / 2} \doteqdot 1.056 \times 10^{-21} \\
& \mu(3) \doteqdot 358044943 \quad 52709118865923233881 \\
& .7178201368 \\
& 2.75(\mu(3))^{-1 / 2} \doteqdot 1.453 \times 10^{-14} \\
& \mu(5) \doteqdot \quad 13563211 \quad 2763154707 \cdot 1271775370 \\
& 2.75(\mu(5))^{-1 / 2} \doteqdot 7.467 \times 10^{-9} \\
& \mu(7) \doteqdot \quad 172 \quad 7435358829 \cdot 8828254344 \\
& 2.75(\mu(7))^{-1 / 2} \doteqdot 2.092 \times 10^{-6} .
\end{aligned}
$$

The remaining calculations shown below have been chopped to the number of digits shown. 


\begin{tabular}{|c|c|c|c|c|}
\hline $\mathbf{r}$ & $\mu(r)$ & $2.75(\mu(r))^{-1 / 2}$ & f & $\boldsymbol{l}$ \\
\hline 11 & $61296758 \cdot 9923$ & $3.512 \times 10^{-4}$ & - & \\
\hline 13 & $3884813 \cdot 7538$ & 0.00139 & - & \\
\hline 17 & 109378.0445 & 0.00831 & - & \\
\hline 19 & 32249.0150 & 0.0153 & 32249 & $-484 \cdot 146$ \\
\hline 23 & $5301 \cdot 840$ & 0.0377 & - & \\
\hline 29 & 899.198 & 0.0917 & - & \\
\hline 31 & 579.808 & 0.114 & - & \\
\hline 37 & 206.625 & 0.191 & - & \\
\hline 41 & $122 \cdot 832$ & 0.248 & 123 & $20 \cdot 609$ \\
\hline 43 & 98.205 & 0.277 & 98 & $-20 \cdot 156$ \\
\hline 47 & 66.464 & 0.337 & - & \\
\hline 53 & $41 \cdot 329$ & 0.427 & 41 & $-13 \cdot 595$ \\
\hline
\end{tabular}

Thus, we conclude that $\mu$ is fundamental.

Department of Mathematics

Massey University

Palmerston North, New Zealand

1. B. D. BEACH, H. C. WILLIAMS \& C. R. ZARNKE, "Some computer results on units in quadratic and cubic fields," Proc. 25th Summer Meeting Canad. Math. Congress, 1971, pp. 609648.

2. MARTA SVED, “Units in pure cubic number fields," Ann. Univ. Sci. Budapest. Eötvös Sect. Math., v. 13, 1970, pp. 141-149.

3. G. SZEKERES, "Multidimensional continued fractions," Ann. Univ. Sci. Budapest. Eötvös Sect. Math., v. 13, 1970, pp. 113-140. 